\title{
DISCRETE NUMERICAL SOLVABILITY OF HAMMER- STEIN INTEGRAL EQUATIONS OF MIXED TYPE
}

\author{
M. GANESH AND M.C. JOSHI
}

ABSTRACT. In a recent paper using a variation of the Kumar and Sloan new collocation-type method, we studied the numerical solvability of Hammerstein integral equation of mixed type

$$
x(s)+\sum_{i=1}^{m} \int_{a}^{b} k_{i}(s, t) f_{i}(t, x(t)) d t=y(s), \quad s \in[a, b] .
$$

In this paper a discretized version of the above method is considered. The discrete version is obtained when the integrals are evaluated using quadrature formula. Using interpolatory quadrature rules and piecewise-polynomial function spaces, the convergence of the discrete approximate solutions to the actual solution of (I) is proved. The order of convergence is obtained when the quadrature rule is of certain degree of precision.

1. Introduction. In [3], we studied the numerical solvability of Hammerstein integral equation of mixed type

$$
x(s)+\sum_{i=1}^{m} \int_{a}^{b} k_{i}(s, t) f_{i}(t, x(t)) d t=y(s), \quad s \in[a, b]
$$

where $-\infty<a<b<\infty, y, k_{i}$ and $f_{i}$ are known functions and $x$ is a solution to be determined.

A discretized version of the numerical solvability discussed in [3] is considered in this paper. The discretized version is obtained when all the definite integrals required to be evaluated for computing the numerical solution of (1.1) using the method of $[\mathbf{3}]$, are approximated by numerical quadrature.

The convergence of the discrete approximate solutions to the actual solution of (1.1) is discussed and its rate of convergence is obtained in certain piecewise-polynomial function spaces. When the quadrature 
rule is of certain degree of precision, the superconvergence rates obtained in $[\mathbf{3}]$ are maintained in the discrete case. The results obtained are extensions of the results of $[\mathbf{5}]$.

2. Preliminaries. Let $C=C[a, b]$ be the Banach space of continuous real valued functions on $[a, b]$ equipped with uniform norm and let $R=R[a, b]$ be the Banach space of bounded Riemann-integrable real-valued functions on $[a, b]$. The following assumptions will be used to carry out the analysis in the spaces $C$ and $R$. Let $x^{*} \in C$ be a solution of (1.1) and

$$
B\left(x^{*}, \delta\right)=\left\{x \in C:\left\|x-x^{*}\right\|_{\infty} \leq \delta\right\} .
$$

Assumptions [A].

A1. $y \in C$; for each $i, 1 \leq i \leq m$,

A2. the kernel $k_{i}(s . t)$ is continuous on $a \leq s, t \leq b$,

A3. the function $f_{i}(t, x)$ is defined and continuous on $[a, b] \times \mathbf{R}$,

A4. the partial derivative $f_{i, x}(t, x)=(\delta / \delta x) f_{i}(t, x)$ exists and is continuous on $[a, b] \times \mathbf{R}$,

A5. the function $f_{i, x}$ satisfies the Lipschitz condition; there exists a constant $\alpha_{i}$ such that

$$
\left|f_{i, x}\left(t, x_{1}(t)\right)-f_{i, x}\left(t, x_{2}(t)\right)\right| \leq \alpha_{i}\left|x_{1}(t)-x_{2}(t)\right|
$$

for all $t \in[a, b]$ and all $x_{1}, x_{2} \in B\left(x^{*}, \delta\right)$ for some $\delta>0$.

For $1 \leq i \leq m$, Assumption A2 implies that each linear integral operator $K_{i}$ defined by

$$
\left[K_{i} x\right](s)=\int_{a}^{b} k_{i}(s, t) x(t) d t, \quad s \in[a, b], \quad x \in R
$$

is a compact operator from $R$ to $C$ and assumption A3 implies that each Nemytskii operator $N_{i}$ defined as

$$
\left[N_{i} x\right](t)=f_{i}(t, x(t)), \quad t \in[a, b], \quad x \in C
$$

is a continuous, bounded operator from $C$ to $C$. 
So (1.1) can be written as an operator equation

$$
x+\sum_{i=1}^{m} K_{i} N_{i} x=y .
$$

Existence and uniqueness of the solution of (2.3) are studied in [3]. As in $[\mathbf{3}]$, we sought the approximation in piecewise-polynomial function spaces which are defined below:

For any natural number $n$, let

$$
\prod_{n}: a=s_{1}<s_{2} \cdots<s_{n}<s_{n+1}=b
$$

be a partition of $[a, b]$ and let

$$
h=h(n)=\max _{1 \leq i \leq n}\left(s_{i+1}-s_{i}\right) .
$$

Assume that $h \rightarrow 0$ as $n \rightarrow \infty$ and the partition $\prod_{n}$ is quasi-uniform, that is there exists a constant $\beta$ such that

$$
h \leq \beta \min _{1 \leq i \leq n}\left(s_{i+1}-s_{i}\right)
$$

With $r$ a positive integer and $\gamma$ an integer satisfying $0 \leq \gamma<r$, let $S_{r, n}^{\gamma}$ denote the space of piecewise-polynomial functions of order $r$ and continuity $\gamma$. That is $\phi \in S_{r, n}^{\gamma}$, if and only if, it is a polynomial of degree $\leq r-1$ on each subinterval $\left(s_{i}, s_{i+1}\right), 1 \leq i \leq n$ and has $\gamma-1$ continuous derivatives on $(a, b)$. If $\gamma=0$ there is no continuity requirement at break points $s_{i}, 1 \leq i \leq n+1$. In this case we arbitrarily take each $\phi \in S_{r, n}^{0}$ to be right continuous at $s_{1}=a$ and left continuous at every $s_{i}, 2 \leq i \leq n+1$.

For $N=(n-1)(r-\gamma)+r$ (the dimension of $\left.S_{r, n}^{\gamma}\right)$, let $\left\{u_{j}\right\}_{j=1}^{n}$ be a basis for $S_{r, n}^{\gamma}$ and let $\left\{\tau_{i}\right\}_{i=1}^{N}$ be a set of distinct points in $[a, b]$ such that $u_{j}\left(\tau_{i}\right)=\delta_{i j}$.

3. Numerical Solvability. In [3], the numerical solution of (1.1), using a variation of new collocation-type method of $[\mathbf{6}]$, is computed as follows. 
First find collocation approximation to the functions $z_{i}, 1 \leq i \leq m$, defined by

$$
z_{i}(s)=f_{i}(s, x(s)), \quad s \in[a, b] .
$$

Substituting (3.1) in (1.1), we have

$$
x(s)=y(s)-\sum_{j=1}^{m} \int_{a}^{b} k_{j}(s, t) z_{j}(t) d t, \quad s \in[a, b]
$$

and (3.1) can be written as

$$
z_{i}(s)=f_{i}\left(s, y(s)-\sum_{j=1}^{m} \int_{a}^{b} k_{j}(s, t) z_{j}(t) d t\right), \quad 1 \leq i \leq m .
$$

The collocation approximation to $z_{i}$ is of the form

$$
z_{i, n}(t)=\sum_{j=1}^{N} a_{i, j} u_{j}(t), \quad 1 \leq i \leq m
$$

where $\left\{u_{j}\right\}_{j=1}^{N}$ is a basis for $S_{r, n}^{\gamma}$ and the coefficients $a_{i}, \ldots, a_{i, N}$, $1 \leq i \leq m$ are determined by collocating (3.3) at the (collocation) points $\tau_{1}, \ldots, \tau_{N}$ :

$$
\begin{gathered}
z_{i, n}\left(\tau_{l}\right)=f_{i}\left(\tau_{l}, y\left(\tau_{l}\right)-\sum_{j=1}^{m} \int_{a}^{b} k_{j}\left(\tau_{l}, t\right) z_{j, n}(t) d t\right), \\
1 \leq i \leq m, \quad 1 \leq l \leq N .
\end{gathered}
$$

That is

$$
\begin{gathered}
z_{i, n}\left(\tau_{l}\right)=f_{i}\left(\tau_{l}, y\left(\tau_{l}\right)-\sum_{j=1}^{m} \sum_{q=1}^{N} a_{j, q} \int_{a}^{b} k_{j}\left(\tau_{l}, t\right) u_{q}(t) d t\right), \\
1 \leq i \leq m, \quad 1 \leq l \leq N .
\end{gathered}
$$

The approximation $x_{n}$ to the solution $x^{*}$ of (1.1) is given by

$$
\begin{aligned}
x_{n}(s) & =y(s)-\sum_{j=1}^{m} \int_{a}^{b} k_{j}(s, t) z_{j}(t) d t \\
& =y(s)-\sum_{j=1}^{m} \sum_{q=1}^{N}\left(\int_{a}^{b} k_{j}(s, t) u_{q}(t) d t\right) a_{j, q} .
\end{aligned}
$$


Note that for $1 \leq i \leq m$, the calculation of $z_{i, n}$ requires the evaluation of the definite integrals $K_{j} u_{q}\left(\tau_{l}\right), 1 \leq j \leq m, 1 \leq q \leq N, 1 \leq l \leq N$. Also the calculation for $x_{n}$ requires the evaluation of the definite integrals $K_{j} u_{q}(s), 1 \leq j \leq m, 1 \leq q \leq N$, for $s \in[a, b]$. When these integrals are approximated by numerical quadrature, we get the discretized version of the above method, which is discussed in the next section.

Let $P_{n}: C+S_{r, n}^{\gamma} \rightarrow S_{r, n}^{\gamma}$ be the interpolatory operator defined by

$$
\left[P_{n} x\right](t)=\sum_{j=1}^{N} x\left(\tau_{j}\right) u_{j}(t), \quad t \in[a, b], \quad x \in C+S_{r, n} .
$$

Assume that the collocation points $\left\{\tau_{i}\right\}_{i=1}^{N}$ are chosen in such a way that $P_{n}$ is uniformly bounded as an operator from $C+S_{r, n}^{\gamma}$ to $S_{r, n}^{\gamma}$, that is

$$
\left\|P_{n}\right\| \leq M
$$

where $M>0$ is independent of $n$ and

$$
\lim _{n \rightarrow \infty}\left\|P_{n} x-x\right\|_{\infty}=0 \text { for all } x \in C .
$$

Using operator theoretic representations, (3.3) and (3.4), together with (3.5), and (3.7) can be written as

$$
z_{i}=N_{i}\left(y-\sum_{j=1}^{m} K_{j} z_{j}\right), \quad 1 \leq i \leq m
$$

$$
z_{i, n}=P_{n} N_{i}\left(y-\sum_{j=1}^{m} K_{j} z_{j, n}\right), \quad 1 \leq i \leq m
$$

and

$$
x_{n}=y-\sum_{j=1}^{m} K_{j} z_{j, n}
$$

respectively. 
Theorem 3.1. [3]. Let Assumptions A1 through A4 hold. Let $x^{*} \in C$ be a solution of (1.1) and the interpolatory operator $P_{n}$ satisfy (3.8) and (3.9). Let $\sum_{i=1}^{m}\left(K_{i}, N_{i}\right)^{\prime}\left(x^{*}\right)$ not have -1 as an eigenvalue. Then, for sufficiently large $n,(3.12)$ has a unique solution $x_{n}$ in $C$ such that $x_{n} \rightarrow x^{*}$ in supremum norm and satisfies the error estimate

$$
\begin{aligned}
M_{2}\left\|\sum_{i=1}^{m} K_{i}\left(P_{n} N_{i} x^{*}-N_{i} x^{*}\right)\right\|_{\infty} & \leq\left\|x_{n}-x^{*}\right\|_{\infty} \\
& \leq M_{1} \sum_{i=1}^{m}\left\|K_{i}\left(P_{n} N_{i} x^{*}-N_{i} x^{*}\right)\right\|_{\infty}
\end{aligned}
$$

4.1 where $M_{1}, M_{2}>0$ are independent of $n$.

4. Discrete solvability. In the discrete method, approximate the integrals $K_{j} u_{q}\left(\tau_{l}\right), 1 \leq j \leq m, 1 \leq q, l \leq N$ in (3.6) and the integrals $K_{j} u_{q}(s), 1 \leq j \leq m, 1 \leq q \leq N, s \in[a, b]$ in (3.7) by numerical quadrature process of $[\mathbf{4}]$.

For $x \in R[0,1]$, let the points $p_{1}, \ldots, P_{L} \in[0,1]$ and the weights $w_{1}, \ldots, w_{L}$ generate the quadrature rule

$$
\int_{0}^{1} x(t) d t \simeq \sum_{j=1}^{L} w_{j} x\left(p_{j}\right)
$$

which is exact for polynomials of degree $\leq \rho$, but not exact for polynomials of degree $\rho+1$ (that is, the quadrature rule has degree of precision $\rho$ ). For $1 \leq i \leq m$, let $K_{i, n}: R \rightarrow C$ be the discrete integral operator defined by

$$
\left[K_{i, n} x\right](t)=\sum_{j=1}^{n} \sum_{q=1}^{L} w_{q} h_{j} k_{i}\left(t, t_{j q}\right) x\left(t_{j q}\right),
$$

where $h_{j}=s_{j+1}-s_{j}$ and $t_{j q}=s_{j}+h_{j} p_{q}$. For $x \in R, K_{i} x$ is approximated by $K_{i, n} x$ by shifting the quadrature rule (4.1) to each subinterval $\left(s_{j}, s_{j+1}\right), 1 \leq j \leq n$ of the partition $\prod_{n}$. Note that the integrals to be approximated in (3.6) and (3.7) are of the form $K_{i} \phi_{n} 1 \leq i \leq m$ where $\phi_{n} \in S_{r, n}^{\gamma}$. The following theorem gives an estimate in the quadrature error, for this case. 
For $1 \leq i \leq m$, let $k_{i}^{s}(t)=k_{i}(s, t)$ for $a \leq s, t \leq b$. For $1 \leq p \leq \infty$, with $l$ a non-negative integer, let $W_{l}^{p}=W_{l}^{p}(a, b)$ be the usual Sobolev space with norm $\|\cdot\|_{l . p}([\mathbf{1}])$.

Theorem 4.1. [4] For $1 \leq i \leq m$, let $k_{i}^{s} \in W_{\mu}^{1}(\mu \geq 1)$ and $\left\|k_{i}^{s}\right\|_{\mu, 1}$ be bounded independently of $s$. Then for all $\phi_{n} \in S_{r, n}^{\gamma}$

$$
\left\|\left(K_{i}-K_{i, n}\right) \phi_{n}\right\| \leq c_{i} h^{\beta} \max _{1 \leq q \leq n}\left[\sum_{j=0}^{\beta}\left[\sup _{s_{q}<t<s_{q+1}}\left|\phi_{n}^{(j)}(t)\right|\right]\right]
$$

where $\beta=\min (\mu, \rho+1)$ and each $c_{i}$ is a constant independent of $n$.

The following lemma gives the properties of $K_{i, n}, 1 \leq i \leq m$.

Lemma 4.1 [2]. For $1 \leq i \leq m$, let Assumption A2 hold. Then for each $i$

(i) $K_{i, n} x \rightarrow K_{i} x$ for all $x \in R$,

(ii) $\left\{K_{i, n}: n \geq 1\right\}$ is collectively compact.

The discrete analogs corresponding to $z_{i, n}$ and $x_{n}$ can be written as operator equations

$$
\tilde{z}_{i, n}=P_{n} N_{i}\left(y-\sum_{j=1}^{m} K_{j, n} \tilde{z}_{j, n}\right), \quad 1 \leq i \leq m
$$

and

$$
\tilde{x}_{n}=y-\sum_{j=1}^{m} K_{j, n} \tilde{z}_{j, n}
$$

respectively.

Let

$$
C_{m}=C_{m}[a, b]=C \times \cdots \times C(m \text { times }),
$$

for $\mathbf{u}=\left(u_{1}, \ldots, u_{m}\right) \in C_{m},\|\mid \mathbf{u}\|_{\infty}=\left(\sum_{i=1}^{m}\left\|u_{i}\right\|_{\infty}^{2}\right)^{1 / 2}$ be the norm in $C_{m}$,

$$
R_{m}=R_{m}[a, b]=R \times \cdots \times R(m \text { times })
$$


and norm in $R_{m}$ be $\||\cdot|\|_{\infty}$ defined in a similar way.

Let $K: R_{m} \rightarrow C_{m}, K_{n}: R_{m} \rightarrow C_{m}$ and $N: C_{m} \rightarrow C_{m}$ be matrix operators defined as follows:

$$
K=\left[\begin{array}{cccc}
K_{1} & K_{2} & \cdots & K_{m} \\
K_{1} & K_{2} & \cdots & K_{m} \\
\cdot & \cdot & \cdots & \cdot \\
\cdot & \cdot & \cdots & \cdot \\
\cdot & \cdot & \cdots & \cdot \\
K_{1} & K_{2} & \cdots & K_{m}
\end{array}\right]
$$

$$
K_{n}=\left[\begin{array}{cccc}
K_{1, n} & K_{2, n} & \cdots & K_{m, n} \\
K_{1, n} & K_{2, n} & \cdots & K_{m, n} \\
\cdot & \cdot & \cdots & \cdot \\
\cdot & \cdot & \cdots & \cdot \\
\cdot & \cdot & \cdots & \cdot \\
K_{1, n} & K_{2, n} & \cdots & K_{m, n}
\end{array}\right]
$$

$$
N=\left[\begin{array}{llll}
N_{1} & & & \\
& N_{2} & & \\
& & \ddots & \\
& & & N_{m}
\end{array}\right]
$$

Since all $K_{i}$ 's, $1 \leq i \leq m$, are compact linear operators from $R$ to $C$, we have that $K$ is also a compact linear and completely continuous operator from $R_{m}$ to $C_{m}$. The properties of $K_{i, n}, 1 \leq i \leq m$, are also carried over to $K_{n}$ and the following lemma gives this.

Lemma 4.2. For $1 \leq i \leq m$, let Assumption A2 hold. Then

(i) $K_{n} \mathbf{u} \rightarrow K \mathbf{u}$ for all $\mathbf{u} \in R_{m}$,

(ii) $\left\{K_{n}: n \geq 1\right\}$ is collectively compact.

Let $T: R_{m} \rightarrow C_{m}$ be defined as

$$
T \mathbf{z}=\mathbf{v}-K \mathbf{z}, \quad \mathbf{z} \in R_{m}
$$


where $\mathbf{v}=(y, \ldots, y) \in C_{m}$ and $K$ is as defined in (4.5). Since $K$ is completely continuous, $T$ is also completely continuous.

Let $T_{n}: R_{m} \rightarrow C_{m}$ be defined as

$$
T_{n} \mathbf{z}=\mathbf{v}-K_{n} \mathbf{z}, \quad \mathbf{z} \in R_{m} .
$$

From Lemma 4.2, it follows that $T_{n} \mathbf{z} \rightarrow T \mathbf{z}$ for all $\mathbf{z} \in \mathbf{R}_{m}$ and $\left\{T_{n}: n \geq 1\right\}$ is collectively compact. Since $N$ is continuous, we have $\left\{N T_{n}: n \geq 1\right\}$ is collectively compact. For $1 \leq i \leq m$ Assumptions $A 3$ and $A 4$ imply that $N_{i}$ is continuously Frechet differentiable on $C$; its Frechet derivative at $x_{0} \in C$ is a bounded linear operator given by

$$
\left[N_{i}^{\prime}\left(x_{0}\right) u\right](t)=f_{i, x}\left(t, x_{0}(t)\right) u(t), \quad t \in[a, b], \quad u \in C .
$$

Let $\delta_{1}=m^{1 / 2} \delta$ and $\mathbf{u}=\left(x^{*}, \ldots, x^{*}\right)$ where $x^{*}$ is a solution of $(1.1)$. Let

$$
B\left(\mathbf{u}, \delta_{1}\right)=\left\{\mathbf{w} \in C_{m}:\|\|_{\infty} \mathbf{u}-\mathbf{w} \|_{\infty} \leq \delta_{1}\right\} .
$$

Lemma 4.3. Let assumptions $[A]$ hold. Then for all $\boldsymbol{\phi}, \boldsymbol{\psi} \in R_{m}$ with $T \boldsymbol{\phi}, T \boldsymbol{\psi} \in B(\mathbf{u}, \delta)$,

$$
\left\|\left|(N T)^{\prime}(\boldsymbol{\phi})-(N T)^{\prime}(\boldsymbol{\psi})\right|\right\|_{\infty} \leq \alpha\left|\|\boldsymbol{\phi}-\boldsymbol{\psi} \mid\|_{\infty}\right.
$$

for some constant $\alpha>0$.

Proof. For each $\mathbf{w}=\left(w_{1}, \ldots, w_{m}\right) \in C_{m}$, the Frechet derivative $N^{\prime}(\mathbf{w})$ is given by

$$
N^{\prime}(\mathbf{w})=\left[\begin{array}{lll}
N_{1}^{\prime}\left(w_{1}\right) & & \\
& \ddots & \\
& & N_{m}^{\prime}\left(w_{m}\right)
\end{array}\right]
$$

Let $\boldsymbol{\phi}, \boldsymbol{\psi} \in R_{m}$ be given by $\boldsymbol{\phi}=\left(\phi_{1}, \ldots, \phi_{m}\right)$ and $\boldsymbol{\psi}=\left(\psi, \ldots, \psi_{m}\right)$. 
Also let $T \boldsymbol{\phi}, T \boldsymbol{\psi} \in B\left(\mathbf{u}, \delta_{1}\right)$. Now

$$
\begin{aligned}
\|\|(N T)^{\prime}(\boldsymbol{\phi})- & (N T)^{\prime}(\boldsymbol{\psi}) \|_{\infty}^{2} \\
& =\|\|\left[N^{\prime}(T \boldsymbol{\phi})-N^{\prime}(T \boldsymbol{\psi})\right] T \|_{\infty}^{2} \\
& \leq\||| T \mid\|^{2} \sum_{i=1}^{m}\left\|N_{1}^{\prime}\left(y-\sum_{j=1}^{m} K_{j} \phi_{j}\right)-N_{i}^{\prime}\left(y-\sum_{j=1}^{m} K_{j} \psi_{j}\right)\right\|_{\infty}^{2} \\
\leq & \|\| T||^{2} \sum_{i=1}^{m} \sup _{a \leq t \leq b} \mid f_{i, x}\left(t, y(t)-\sum_{j=1}^{m} K_{j} \phi_{j}(t)\right) \\
& -\left.f_{i, x}\left(t, y(t)-\sum_{j=1}^{m} k_{j} \psi_{j}(t)\right)\right|^{2} .
\end{aligned}
$$

Since $\mathbf{u}=\left(x^{*}, \ldots, x^{*}\right)$ and $T \mathbf{w}=\left(y-\sum_{j=1}^{m} K_{j} w_{j}, \ldots, y-\right.$ $\left.\sum_{j=1}^{m} k_{j} w_{j}\right)$, for any $\mathbf{w} \in R_{m}$ given by $\mathbf{w}=\left(w_{1}, \ldots, w_{m}\right)$, we have $\left(y-\sum_{j=1}^{m} K_{j} \phi_{j}\right)$ and $\left(y-\sum_{j=1}^{m} K_{j} \psi_{j}\right) \in B\left(x^{*}, \delta\right)$ and using Assumption $\mathrm{A} 5$, we get

$$
\begin{aligned}
\| \mid(N T)^{\prime}(\boldsymbol{\phi})- & (N T)^{\prime}(\boldsymbol{\psi}) \|_{\infty}^{2} \\
& \leq\||| T\|_{\infty}^{2}\left(\sum_{i=1}^{m} \alpha_{i}^{2}\right) \sup _{a \leq t \leq b}\left|\sum_{j=1}^{m} K_{j}\left(\phi_{j}(t)-\psi_{j}(t)\right)\right|^{2} \\
& \leq\||| T\|_{\infty}^{2}\left(\sum_{i=1}^{m} \alpha_{i}^{2}\right)\left(\max _{1 \leq j \leq m}\left\|K_{j}\right\|^{2}\right) \sum_{j=1}^{m} \sup _{a \leq t \leq b}\left|\phi_{j}(t)-\psi_{j}(t)\right|^{2} .
\end{aligned}
$$

Assumption A2 implies $\||T|\|<\infty,\left\|K_{j}\right\|<\infty$ for $1 \leq j \leq m$, let $\alpha^{2}=\left\||\|T\||^{2}\left(\sum_{i=1}^{m} \alpha_{i}^{2}\right)\left(\max _{1 \leq j \leq m}\left\|K_{j}\right\|^{2}\right)\right.$. So we have

$$
\left\|\mid(N T)^{\prime}(\phi)-(N T)^{\prime}(\boldsymbol{\psi})\right\|_{\infty}^{2} \leq \alpha^{2} \sum_{j=1}^{m}\left\|\phi_{j}-\psi_{j}\right\|_{\infty}^{2} .
$$

This implies $\left\|\mid(N T)^{\prime}(\phi)-(N T)^{\prime}(\boldsymbol{\psi})\right\|_{\infty} \leq \alpha\|\| \phi-\boldsymbol{\psi} \|_{\infty}$ and the lemma is proved. $\square$

REMARK 4.1. Lemma 4.3 holds, even if we replace $T$ by $T_{n}$. 
Lemma 4.4. [3]. Let Assumptions A1 through A4 hold. Let $x^{*} \in C$ be a solution of (1.1). Let -1 be not an eigenvalue of $\sum_{i=1}^{m}\left(K_{i} N_{i}\right)^{\prime}\left(x^{*}\right)$. Then $(N T)^{\prime}(N \mathbf{u})\left(\mathbf{u}=\left(x^{*}, \ldots, x^{*}\right)\right)$ exists and does not have 1 as an eigenvalue.

The following theorem gives the convergence of $\tilde{x}_{n}$ to $x^{*}$.

Theorem 4.2. Let Assumptions $[A]$ hold. Let $x^{*} \in C$ be a solution of (1.1) and the interpolatory operator $P_{n}$ satisfy (3.8) and (3.9). Let $\sum_{i=1}^{m}\left(K_{i} N_{i}\right)^{\prime}\left(x^{*}\right)$ not have -1 as an eigenvalue. Then for sufficiently large $n,(4.4)$ has a unique solution $\tilde{x}_{n}$ in $C$ such that

$$
\left\|\tilde{x}_{n}-x^{*}\right\|_{\infty} \rightarrow 0 \text { as } n \rightarrow \infty \text {. }
$$

Proof. It is easy to see that $\mathbf{u}=\left(x^{*}, \ldots, x^{*}\right)$ is a solution of the equation

$$
\mathbf{u}=T N \mathbf{u}
$$

The system of equations (3.10) can be written as

$$
\mathbf{z}=N T \mathbf{z}
$$

in the product space. Since $\mathbf{u}$ is a solution of (4.8), we have that $\mathbf{z}=N \mathbf{u}\left(N_{1} x^{*}, \ldots, N_{m} x^{*}\right)$ is the solution of (4.9). Let

$$
\begin{aligned}
& \chi_{1}=\left(C+S_{r, n}^{\gamma}\right) \times \cdots \times\left(C+S_{r, n}^{\gamma}\right) \quad(m \text { times }), \\
& \chi_{2}=S_{r, n}^{\gamma} \times \cdots \times S_{r, n}^{\gamma} \quad(m \text { times }) .
\end{aligned}
$$

Let $\mathbf{P}_{n}: \chi_{1} \rightarrow \chi_{2}$ be defined by

$$
\mathbf{P}_{n}=\left[\begin{array}{llll}
P_{n} & & & \\
& P_{n} & & \\
& & \ddots & \\
& & & P_{n}
\end{array}\right]
$$

Since $P_{n}$ is a projection, we have that $\mathbf{P}_{n}$ is also a projection. (3.8) implies that $\mathbf{P}_{n}$ is uniformly bounded as an operator from $\chi_{1}$ to $\chi_{2}$, that is

$$
\left\|\left|\mathbf{P}_{n} \|\right| \leq M_{3}\right.
$$


(3.9) implies that

$$
\lim _{n \rightarrow \infty}\left\|\mathbf{P}_{n} \mathbf{w}-\mathbf{w}\right\|_{\infty}=0 \text { for all } \mathbf{w} \in C_{m} .
$$

Using matrix operators, the system of equations (4.3) can be written as

$$
\tilde{\mathbf{z}}_{n}=\mathbf{P}_{n} N T_{n} \tilde{\mathbf{z}}_{n} .
$$

For the solvability of (4.4), first investigate the solvability of (4.12). For this verify the conditions of Theorem 2 of $[8]$, which is a modification of Theorem 1 of [10]. Lemma 4.4 gives that $(N T)^{\prime}(N \mathbf{u})$ does not have 1 as an eigenvalue. As $(N T)^{\prime}(N u)$ is compact linear, we have that $\left[I-(N T)^{\prime}(N \mathbf{u})\right]$ is nonsingular. Since $\left\{N T_{n}: n \geq 1\right\}$ is collectively compact on $R$, using (4.11) it can be proved (on the lines of Lemma 4 of [5]) that for all $\sigma>0, \sigma \in \mathbf{R}$,

$$
\sup _{w \in B_{\sigma}}\left\|N T_{n}(\mathbf{w})-\mathbf{P}_{n} N T_{n}(\mathbf{w}) \mid\right\|_{\infty} \rightarrow 0 \text { as } n \rightarrow \infty
$$

where $B_{\sigma}=\left\{\mathbf{w}: \mathbf{w} \in R_{m}, \quad\|\mathbf{w}\| \leq \sigma\right\}$. Hence from the Appendix of [5], it follows that $\left\{\mathbf{P}_{n} N T_{n}: n \geq 1\right\}$ is collectively compact on $R$. Moreover for $\mathbf{w} \in R_{m}$,

$$
\begin{aligned}
& \left\|\mathbf{P}_{n} N T_{n} \mathbf{w}-N T \mathbf{w}\right\|_{\infty} \\
& \leq\left\|\mathbf{P}_{n} N T_{n} \mathbf{w}-\mathbf{P}_{n} N T \mathbf{w}\right\|_{\infty}+\left\|\mid \mathbf{P}_{n} N T \mathbf{w}-N T \mathbf{w}\right\|_{\infty} .
\end{aligned}
$$

Since $T_{n} \mathbf{w} \rightarrow T \mathbf{w}, N$ is continuous, $N T_{n} \mathbf{w} \rightarrow N T \mathbf{w}$. Using this, (4.10) and (4.11) in (4.13), $\mathbf{P}_{n} N T_{n} \mathbf{w} \rightarrow N T \mathbf{w}$.

Since for all $\phi \in R_{m}, T_{n} \phi \rightarrow T \phi$ and since $\mathbf{u}$ is a solution of (4.8), there exists $\delta^{*}>0$ such that for all $\phi, \psi \in B\left(\mathbf{z}, \delta^{*}\right), T_{n} \phi, T_{n} \boldsymbol{\psi} \in$ $B\left(\mathbf{u}, \delta_{1}\right)$. Using Lemma 3.1 (with $T$ replaced by $T_{n}$ ) and (4.10), for all $\boldsymbol{\phi}, \boldsymbol{\psi} \in B\left(\mathbf{z}, \delta^{*}\right)$,

$$
\left\|\left|\left(\mathbf{P}_{n} N T_{n}\right)^{\prime}(\phi)-\left(\mathbf{P}_{n} N T_{n}\right)^{\prime}(\psi)\right|\right\|_{\infty} \leq \alpha^{*} \mid\|\phi-\psi\| \|_{\infty}
$$

where $\alpha^{*}=M_{3} \alpha$. Hence all the conditions of Theorem 2 of $[8]$ are satisfied and for sufficiently large $n,(4.12)$ has a unique solution $\tilde{\mathbf{z}}_{n}$ such that $\left\|\mid \tilde{\mathbf{z}}_{n}-\mathbf{z}\right\| \|_{\infty} \rightarrow 0$ as $n \rightarrow \infty$. 
Let $\tilde{\mathbf{z}}_{n}=\left(\tilde{z}_{1, n}, \ldots, \tilde{z}_{m, n}\right)$ and $\mathbf{z}=\left(z_{1}, \ldots, z_{m}\right)$. Now (4.4) is equivalent to the equation

$$
\tilde{\mathbf{u}}_{n}=T_{n} \tilde{\mathbf{z}}_{n}
$$

where $\tilde{\mathbf{z}}_{n}$ is a solution of (4.12). For if $\tilde{\mathbf{u}}_{n}=\left(\tilde{x}_{1, n}, \ldots, \tilde{x}_{m, n}\right)$ is a solution of (4.14) then we have $\tilde{x}_{1, n}=\cdots=\tilde{x}_{m, n}=\tilde{x}_{n}$ and $\tilde{x}_{n}$ is a solution of (4.4). Similarly if $\tilde{x}_{n}$ is a solution of (4.4) then $\tilde{\mathbf{u}}_{n}=\left(\tilde{x}_{n}, \ldots, \tilde{x}_{n}\right)$ is a solution of (4.14). Since $\mathbf{z}_{n}$ is a unique solution of (4.12), $\tilde{\mathbf{u}}_{n}=T_{n} \tilde{\mathbf{z}}_{n}$ is a unique solution of (4.14). So $\tilde{\mathbf{u}}_{n}$ will be of the form $\tilde{\mathbf{u}}_{n}=\left(\tilde{x}_{n}, \ldots, \tilde{x}_{n}\right)$ and $\tilde{x}_{n}$ is a unique solution of (4.4). Now (4.15)

$$
\begin{aligned}
\left\|\tilde{\mathbf{u}}_{n}-\mathbf{u}\right\|_{\infty} & =\left\|\mid T_{n} \tilde{\mathbf{z}}_{n}-T \mathbf{z}\right\| \|_{\infty} \\
& =m^{1 / 2} \sum_{i=1}^{m}\left\|K_{n, i} \tilde{z}_{i, n}-K_{i} z_{i}\right\|_{\infty} \\
& \leq m^{1 / 2} \sum_{i=1}^{m}\left[\left\|K_{n, i} \tilde{z}_{i, n}-K_{n, i} z_{i}\right\|_{\infty}+\left\|K_{n, i} z_{i}-K_{i} z_{i}\right\|_{\infty}\right] \\
& \leq m^{1 / 2} \sum_{i=1}^{m}\left[\left\|K_{n, i}\right\|\left\|\tilde{z}_{i, n}-z_{i}\right\|_{\infty}+\left\|K_{n, i} z_{i}-K_{i} z_{i}\right\|_{\infty}\right] .
\end{aligned}
$$

$\left\|\tilde{\mathbf{z}}_{n}-\mathbf{z}\right\|_{\infty} \rightarrow 0$ as $n \rightarrow \infty$ implies that $\left\|\tilde{z}_{i, n}-z_{i}\right\|_{\infty} \rightarrow 0$ as $n \rightarrow \infty, 1 \leq i \leq m$. From Lemma 4.1, $\left\|K_{n, i} z_{i}-K_{i} z_{i}\right\|_{\infty} \rightarrow 0$ as $n \rightarrow \infty, 1 \leq i \leq m$. Using these and uniform boundedness principle in (4.15), \|\|$\tilde{\mathbf{u}}_{n}-\mathbf{u} \|_{\infty} \rightarrow 0$ as $n \rightarrow \infty$. Since

$$
\left\|\tilde{\mathbf{u}}_{n}-\mathbf{u}\right\|_{\infty}=m^{1 / 2}\left\|\tilde{x}_{n}-x\right\|_{\infty},
$$

we have $\left\|\tilde{x}_{n}-x\right\| \rightarrow 0$ as $n \rightarrow \infty$.

As in [3], for $\gamma=0$ and $\gamma=1$, we give the order of convergence (referred as superconvergence) of $\tilde{x}_{n}$ to $x$. Also, it can be shown that when the kernels are sufficiently smooth, $\tilde{z}_{i, n}, 1 \leq i \leq m$, will exhibit up to $\mathrm{O}\left(h^{r}\right)$ convergence. But for $\gamma=0$ and $\gamma=1$ and for particular sets of collocation points, $\tilde{x}_{n}$ will exhibit a better rate of convergence, known as superconvergence. The sets of collocation points chosen for this purpose are as follows. 
Case $\gamma=0$. The dimension of $S_{r, n}^{0}$ is $N=n r$ and we need $n r$ collocation points. Let $\eta_{1}, \ldots, \eta_{r}$ be the zeros of the $r$ th degree Legendre polynomial $\phi_{r}(s), s \in[-1,1]$, which are known as Gauss-Legendre points. The collocation points $\left\{\tau_{j}\right\}_{j=1}^{N}$ are the points $\eta_{1}, \ldots, \eta_{r}$ linearly shifted to each subinterval $\left(s_{i}, s_{i+1}\right), 1 \leq i \leq n$ :

$$
\tau_{(i-1) r+j}=\left[s_{i}+s_{i+1}+\left(s_{i+1}-s_{i}\right) \eta_{j}\right] / 2, \quad 1 \leq j \leq r, \quad 1 \leq i \leq n .
$$

Case $\gamma=1$. In this case $r$ is necessarily $\geq 2$ as the collocation approximation is sought in $S_{r . n}^{1}$, the space of continuous piecewisepolynomial functions. As the dimension of $S_{r . n}^{1}$ is $N=n r-n+1$, we need $n r-n+1$ collocation points. Let $\eta_{1}, \ldots, \eta_{r-2}$ be the zeros of $\left.\phi_{r-1}^{(1)}\right)(s), r \geq 3$ (the first derivative of $\phi_{r-1}(s)$ ), which are known as Lobatto points. Let $\eta_{r-1}=1$. The collocation points $\left\{\tau_{j}\right\}_{j=1}^{N}$ are the break points $s_{i}, 1 \leq i \leq n+1$, plus $\eta_{1}, \ldots, \eta_{r-1}$ shifted linearly to each subinterval $\left(s_{i}, s_{i+1}\right), 1 \leq i \leq n$ :

$$
\begin{gathered}
\tau_{(i-1)(r-1)+j+1}=\left[s_{i}+s_{i+1}+\left(s_{i+1}-s_{i}\right) \eta_{j}\right] / 2 \\
1 \leq j \leq r-1, \quad 1 \leq i \leq n
\end{gathered}
$$

with $\tau_{1}=s_{1}=a$.

For the above sets of collocation points, $P_{n}$ will satisfy (3.8) and (3.9) $([\mathbf{9}])$. Using the above sets of collocation points, we have the following rate of convergence of $\tilde{x}_{n}$ to $x$.

TheOREM 4.3. Let $x^{*} \in C$ be a solution of (1.1), let Assumptions $[A]$ hold and let -1 not be an eigenvalue of $\sum_{i=1}^{m}\left(K_{i} N_{i}\right)^{\prime}\left(x^{*}\right)$. For $1 \leq i \leq m$, let $N_{i} x^{*} W_{\mu}^{1}, \mu \geq 1$ and let $k_{i}^{s} \in W_{\beta}^{1} \geq 1$ with $\left\|k_{i}^{s}\right\|_{\beta, 1}$ bounded independently of $s$. Let $\xi_{\gamma}=\min (\mu, \beta, 2 r-2 \gamma), \quad \gamma=0$ or 1 and let $\rho \geq \xi_{\gamma}-1$. Then for sufficiently large $n$, the discrete approximation satisfies

$$
\left\|\tilde{x}_{n}-x^{*}\right\|_{\infty}=\mathrm{O}\left(h \xi_{\gamma}\right)
$$


ProOF.

$$
\begin{aligned}
\left\|x^{*}-\tilde{x}_{n}\right\|_{\infty} \leq & \left\|x^{*}-x_{n}\right\|_{\infty}+\left\|x_{n}-\tilde{x}_{n}\right\|_{\infty} \\
\leq & \left\|x^{*}-x_{n}\right\|_{\infty}+\sum_{i=1}^{m}\left\|K_{i} z_{i, n}-K_{i, n} \tilde{z}_{i, n}\right\|_{\infty} \\
\leq & \left\|x^{*}-x_{n}\right\|_{\infty}+\sum_{i=1}^{m}\left\|K_{i}\left(z_{i, n}-\tilde{z}_{i, n}\right)\right\|_{\infty} \\
& \quad+\sum_{i=1}^{m}\left\|\left(K_{i}-K_{i, n}\right) \tilde{z}_{i, n}\right\|_{\infty} \\
\leq & \left\|x^{*}-x_{n}\right\|_{\infty}+\sum_{i=1}^{m}\left\|K_{i}\right\| \|_{\left(K_{i}-K_{i, n}\right) z_{i, n} \|_{\infty}} \\
& \quad+\sum_{i=1}^{m}\left\|\left(K_{i}-K_{i, n}\right) \tilde{z}_{i, n}\right\|_{\infty} \\
\leq & \mathrm{O}\left(h \xi_{\gamma}\right)
\end{aligned}
$$

where the penultimate step follows from [5] and the last step follows from $[3]$ and $[4]$.

REMARK 4.1. From the above theorem, if $\mu>r$ and $\beta>r$ then $\tilde{x}_{n}$ will exhibit a better rate of convergence than $\tilde{z}_{n}$. We do not have a better rate of convergence for the case $\gamma=1$ and $r=2$. If $\xi_{\gamma}=2 r-2 \gamma$ then $\tilde{x}_{n}$ exhibit up to $\mathrm{O}\left(h^{2 r-2 \gamma}\right)$ (super) convergence while $\tilde{z}_{n}$ exhibit up to $\mathrm{O}\left(h^{r}\right)$ convergence. When $\beta>\min (\mu, 2 r-2 \gamma)$, the rate of convergence of $\tilde{x}_{n}$ to $x$ is the same as that of $x_{n}$ to $x$ given in $[3]$.

REMARK 4.2. Results of [5] can be obtained as corollaries by taking $m=1$.

5. Numerical Example. Consider the mixed Hammerstein integral equation

$$
x(s)+\int_{0}^{1} e^{-(s+t)}[x(t)]^{2} d t+\int_{0}^{1} s t \sin x(t) d t=y(s)
$$


where $y$ is chosen in such as way that $x^{*}(t)=t$ is a solution of (5.1). In this case $y$ is given by

$$
y(s)=s+c_{1} e^{-s}+c_{2} s
$$

where

$$
c_{1}=\int_{0}^{1} e^{-t} t^{2} d t, \quad c_{2}=\int_{0}^{1} t \sin t d t
$$

Discrete approximate solutions for (5.1) with $y$ given by (5.2) are computed using piecewise-quadratic functions with break points $s_{i}=$ $(i-1) / n, i=1, \ldots, n+1$.

According to Theorem 4.3, we can obtain $\mathrm{O}\left(h^{4}\right)$ convergence rate by using an interpolatory quadrature rule with degree of precision $\rho \geq 3$. To see the importance of the degree of precision of quadrature required in Theorem 4.3, discrete approximate solutions are computed with $\rho=1,2$ and 3. Tables 1, 2 and 3 give the estimated order of convergence (EOC) obtained by using Gauss 1-point rule $(\rho=1)$, Ralston 1-point rule $(\rho=2)$ and Gauss 2-point rule $(\rho=3)$ respectively. Tables 1 and 2 clearly indicate that the order of convergence obtained in Theorem 4.3 is not maintained once we use quadrature rules of less degree of precisions than required in Theorem 4.3.

The nonlinear algebraic system of equations are solved by using the subroutine BRENTM [7]. All computations are carried out in double precision on CYBER $180 / 840$ computer. $\left\|x^{*}-\tilde{x}_{n}\right\|$ is estimated by taking the largest of the computed errors at $t_{i}=(i-1) / 250, i=$ $1, \ldots, 251$.

TABLE 1.

$$
(\rho=1)
$$

\begin{tabular}{|rcc|}
\hline \multicolumn{1}{|r}{$n$} & $\left\|x^{*}-\tilde{x}_{n}\right\|$ & $\mathrm{EOC}$ \\
\hline 5 & $1.8650 \mathrm{E}-03$ & 2.0022747 \\
10 & $4.6551 \mathrm{E}-04$ & 2.0007134 \\
15 & $2.0683 \mathrm{E}-04$ & 2.0003512 \\
20 & $1.1633 \mathrm{E}-04$ & 2.0002094 \\
25 & $7.4450 \mathrm{E}-05$ & 2.0001392 \\
30 & $5.1700 \mathrm{E}-05$ & 2.0000992 \\
35 & $3.7983 \mathrm{E}-05$ & \\
\hline
\end{tabular}


TABLE 2 .

\begin{tabular}{|rcc|}
\multicolumn{4}{c}{$(\rho=2)$} \\
\hline$n$ & $\left\|x^{*}-\tilde{x}_{n}\right\|$ & EOC \\
\hline 5 & $1.0196 \mathrm{E}-04$ & 2.9853579 \\
10 & $1.2875 \mathrm{E}-05$ & 2.9929956 \\
15 & $3.8257 \mathrm{E}-06$ & 2.9954295 \\
20 & $1.6161 \mathrm{E}-06$ & 2.9966173 \\
25 & $8.2807 \mathrm{E}-07$ & 2.9973184 \\
30 & $4.7944 \mathrm{E}-07$ & 2.9977802 \\
35 & $3.0202 \mathrm{E}-07$ & \\
\hline
\end{tabular}

TABLE 3 .

\begin{tabular}{|rcc|}
\multicolumn{4}{c}{$(\rho=3)$} \\
\hline$n$ & $\left\|x^{*}-\tilde{x}_{n}\right\|$ & EOC \\
\hline 5 & $1.6376 \mathrm{E}-06$ & 4.0008060 \\
10 & $1.0229 \mathrm{E}-07$ & 4.0002534 \\
15 & $2.0204 \mathrm{E}-08$ & 4.0001248 \\
20 & $6.3927 \mathrm{E}-09$ & 4.0000745 \\
25 & $2.6184 \mathrm{E}-09$ & 4.0000495 \\
30 & $1.2627 \mathrm{E}-09$ & 4.0000354 \\
35 & $6.8158 \mathrm{E}-10$ & \\
\hline
\end{tabular}

\section{REFERENCES}

1. Agmon, S., Lectures on Elliptic Boundary Value Problems, Van Nostrand, New York, 1965.

2. Anselone, P.M. Collectively Compact Operator Approximation Theory, Prentice-Hall, 1971.

3. Ganesh, M. and Joshi, M.C., Numerical solvability of Hammerstein integral equationsof mixed type, Communicated.

4. Joe, S., Discrete collocation methods for second kind Fredholm integral equations, SIAM J. Numer. Anal., 22 (6), (1985), 1167-1177.

5. Kumar, S., A discrete collocation-type method for Hammerstein equations, SIAM J. Numer. Anal., 25 (2), (1988), 328-341.

6. - and Sloan, I.H., A new collocation-type method for Hammerstein equations, Math. Comput. 178 (48), (1987), 585-593.

7. More, J.J. and Cosnard, M.Y., ALGORITHM 554: BRENTM, a Fortran subroutine for the numerical solution of systems of nonlinear equations, ACM Trans. Math. Software, 6, (1980), 240-251.

8. Spence, A. and Moore, G., "A convergence analysis for turning points 
of nonlinear compact operator equations", in Numerical Treatment of Integral Equations, Albrecht, J., and Collatz, L., eds., ISNM 53, Birkhauser Verlag, Basel, 203-212.

9. Vainikko, G. and Uba, P., A piecewise polynomial approximation to the solution of an integral equation with weakly singular kernel, J. Austral. Math. Soc., Ser. B., 22, (1981), 431-438.

10. Weiss, R., On the approximation of fixed points of non-linear compact operators, SIAM J. Numer. Anal., 11, (1974), 550-553.

Department of mathematics, Indian Institute of Technology, Powai, BOMBAY 400 076, India. 